\title{
OBJETOS "MISCELÁNEOS” Y DINÁMICAS SOCIALES EN CONTEXTOS CAZADORES RECOLECTORES DE LA PRECORDILLERA DE ARICA, EXTREMO NORTE DE CHILE
}

\author{
“MISCELLANEOUS” OBJECTS AND SOCIAL DYNAMICS IN \\ HUNTER-GATHERER CONTEXTS IN ARICA FOOTHILLS, \\ NORTHERN CHILE
}

\author{
Camila Castillo 1 y Marcela Sepúlveda²
}

\begin{abstract}
Los patrones de movilidad de los cazadores recolectores altoandinos arcaicos (10.500-3.700 a.p.) del extremo norte de Chile han sido comúnmente definidos a partir de la caracterización y comparación del registro lítico y en menor medida, las pinturas rupestres presentes en aleros o abrigos rocosos. Los demás registros materiales han sido generalmente mencionados de forma ocasional y anecdótica. Trabajos efectuados desde 2006 en adelante, en las tierras altas de la región, han buscado abordar otras dinámicas sociales de estos cazadores recolectores más allá de su subsistencia para así discutir otras esferas de la movilidad de estos grupos. Esta investigación exploratoria se basa en la sistematización cronológica y contextual de objetos ornamentales y/o suntuarios arcaicos, de procedencia local o alóctona, de la precordillera del extremo norte de Chile (2.800-3.800 msm). La presencia de estos objetos, usualmente catalogados como "misceláneos", hallados en diez sitios con ocupaciones arcaicas, nos permiten discutir justamente la movilidad de los grupos altoandinos y su interacción con otros grupos especializados. Asimismo, permiten discutir su rol y relevancia en la conformación de variadas formas de interacción y diferenciación al interior de los grupos. Finalmente, se discute la configuración y mantenimiento de interacciones sociales materializadas por la circulación de objetos misceláneos, y el necesario flujo de información en las dinámicas sociales establecidas entre los períodos Arcaico y Formativo en la región de los Andes Centro-Sur.
\end{abstract}

Palabras claves: objetos “misceláneos”, tierras altas, norte de Chile, período Arcaico, movilidad, redes de interacción.

The mobility patterns of Archaic hunter-gatherers (10,500-3,700 BP) from the highlands of northern Chile have been commonly defined based on the characterization and comparison of lithic materials and, to a lesser extent, on the study of rock art painting found in caves and rock shelters. Other archaeological records have been mentioned occasionally and anecdotally. Investigations carried out from 2006 onwards in the highlands of this region, have sought to address other social dynamics of these hunter-gatherer groups beyond their subsistence, in order to discuss other aspects of their mobility. In this exploratory investigation, we focus on the chronological and contextual systematization of archaic ornamental and sumptuary objects, of local or allochthonous provenance, in the Precordillera (foothills) of northern Chile (2,800-3,800 masl). The presence of these objects, usually classified as "miscellaneous", found in ten sites with archaic occupations, allows us to account for the different mobility patterns of the highland Andean groups and their possible interaction with other specialized groups. They also allow us to discuss their role and importance in the shaping of different interaction and differentiation forms between individuals inside highland groups. Finally, we discuss the configuration and maintenance of social interactions materialized by the circulation of miscellaneous objects, and the necessary flow of information in the social dynamics established between the Archaic and Formative periods in the Central-South Andes region.

Key words: "Miscellaneous" objects, highland, Northern Chile, Archaic Period, mobility, interaction network.

Las sociedades cazadoras recolectoras del Holoceno han sido usualmente descritas como grupos humanos de baja demografía, con una subsistencia basada en un modelo extractivo y una alta movilidad (Bargatzky 1984; Lee y DeVore 1968). Sin embargo, nuevas posturas han ampliado esta visión restringida de la relación ser humano-medio ambiente, para abordar otras dinámicas sociales de estos grupos, a partir de sus interacciones y el flujo de información ocurrido, materializado por ejemplo en la elaboración, uso e intercambio de ciertos objetos (Aldenderfer 1989 y 1999; Aubry et al. 2012; Ericksen 2002; Fitzhugh et al. 2011; Hamilton et al. 2007; Reynolds et al. 2003; Schortman y Urban 2012; Webb 1974; Whallon 2006; Wiessner 1982). En las tierras altas del área Centro-Sur Andina diversas evidencias materiales, tales como las tradiciones o tipologías líticas compartidas entre distintas localidades,

1 Programa de Doctorado en Antropología UCN-UTA, Universidad de Tarapacá, Arica, Chile. camilapaz.f@ me.com
2 Instituto de Alta Investigación, Universidad de Tarapacá, Antofagasta 1520, Arica, Chile. marcelaasre@ gmail.com

Recibido: abril 2016. Aceptado: diciembre 2016.

http://dx.doi.org/10.4067/S0717-73562017005000004. Publicado en línea: 15-marzo-2017. 
las similitudes en el arte rupestre y el hallazgo de objetos categorizados como "misceláneos", al ser objetos no exclusivamente relacionados con el consumo alimenticio y la provisión energética, han permitido discutir la existencia de variadas dinámicas sociales ocurridas en tiempos arcaicos (10.500-3.700 años a.p.), más allá de las prácticas ligadas a la movilidad para la subsistencia (Aldenderfer 1989, 1998 y 2008; Craig 2012; Klink y Aldenderfer 2005; Osorio et al. 2017; Santoro 1989 y 1993; Sepúlveda, García et al. 2013; entre otros). Entre estos objetos "misceláneos" se consideran objetos acabados o materias primas en distintas etapas de procesamiento, de procedencia alóctona a los pisos altoandinos donde fueron hallados, como por ejemplo los fragmentos de valvas del litoral. Estos objetos han sido recurrentemente argüidos para discutir los patrones de movilidad vertical, las posibles relaciones de intercambio e interacción, y consecuentemente la construcción de una historia común entre los distintos grupos arcaicos del área Centro Sur andino (Aldenderfer 1989, 1998, 1999 y 2008; Aldenderfer y Flores 2011; Castillo 2012; Dillehay y Kaulicke 2007; Klink y Aldenderfer 2005; Lavallée et al. 2011; Núñez y Dillehay 1995; Núñez y Santoro 2011; Osorio et al. 2016; Santoro 1993; Schiappacasse y Niemeyer 1975; Sepúlveda, García et al. 2013; Standen 2001; Standen y Núñez 1984; entre otros). Asimismo, con la categoría de "misceláneos", en ciertos casos también llamados "no-utilitarios", se ha hecho referencia a objetos ornamentales y/o suntuarios, es decir objetos cuya funcionalidad no se restringe a la esfera de subsistencia de los cazadores recolectores (Eriksen 2002; Lam 2012; Webb 1974; Whallon 2006). En resumen, la categoría "misceláneos" pareciera agrupar todos aquellos elementos, artefactos y ecofactos, que no evidencian una funcionalidad tecnoeconómica precisa o relacionada exclusivamente con la subsistencia de estos grupos, razón por la cual son comúnmente relegados a un rol secundario en estos contextos de cazadores recolectores. Cuando en realidad resultan indisociables de sus prácticas cotidianas (Ericksen 2002), pues las materias primas empleadas para su producción corresponden a productos secundarios de la caza (materiales óseos, por ejemplo) o recolección (material malacológico, por ejemplo), por lo que su producción nos informa también de la relación humano-medio ambiente (Conneller 2004). Adicionalmente, el hallazgo de estos "misceláneos" nos amplía la mirada sobre la valoración otorgada a estos productos secundarios $\mathrm{y}$, por ende, a esos objetos. Frente a la amplitud del concepto, para efectos de este trabajo consideraremos como "misceláneos" a los objetos completos o incompletos, ornamentales y/o suntuarios, y las materias primas relacionadas con la producción de este tipo de objetos, de origen local o foráneo en relación a la zona estudiada.

Para los cazadores recolectores altoandinos del norte de Chile, los estudios realizados desde la década de 1970 permitieron el análisis del material lítico (tipologías o materias primas) y acentuar la existencia de una movilidad o interacción vertical, sostenida además en la estacionalidad de los recursos en los distintos pisos ecológicos definidos a partir de la gradiente altitudinal (costa, depresión intermedia, pre puna o precordillera y puna) (Lynch 1975; Muñoz y Arriaza 2006; Núñez 1975; Núñez y Dillehay 1995; Núñez y Santoro 1988; Núñez, Zlatar y Núñez 1975; Santoro 1989, 1993; Schiappacasse y Niemeyer 1975; Standen y Núñez 1984; entre otros). Los distintos modelos propuestos han privilegiado desplazamientos entre diversos pisos ecológicos, a nivel amplio, entre la costa y los pisos altoandinos, y más restringidamente entre la costa y los valles bajos, o entre los valles precordilleranos y el altiplano. Si bien estos modelos destacan distintas formas, direccionalidades y temporalidades de los desplazamientos, a su vez han enfatizado la historia y desarrollo de entidades sociales grupales diferenciadas definidas por un determinado modo de subsistencia, asentamiento y tecnologías específicas, en relación con el uso y consumo de recursos complementarios, ofrecidos por los distintos pisos ecológicos distribuidos en la gradiente altitudinal. En particular, dos entidades sociales han sido definidas desde las primeras ocupaciones o primeros poblamientos identificados en la región: los cazadores recolectores altoandinos y los cazadores recolectores y pescadores costeros (Santoro et al. 2016).

En complemento a estos modelos, en las tierras altas del extremo norte de Chile, y tal como fuera planteado previamente por Schiappacasse y Niemeyer (1975), hemos sostenido a partir de estudios sobre el arte rupestre pintado de la precordillera de Arica (Sepúlveda 2011; Sepúlveda, García et al. 2013; Sepúlveda et al. 2017), la evidencia de desplazamientos horizontales, a lo largo de esta franja serrana que se prolonga en el sur del Perú. Basado en las semejanzas estilísticas de 
estas representaciones definidas como "Tradición Naturalista" planteamos que, a partir de finales del Arcaico Medio, hace aproximadamente 6.000 años, aunque con mayor evidencia durante la fase Tardío de este período, ocurriría la construcción de un paisaje cultural compartido por distintos grupos cazadores recolectores de tierras altas (Sepúlveda, García et al. 2013). Sumado a los antecedentes previos, estas nuevas evidencias nos permiten sostener que la movilidad de los grupos altoandinos arcaicos de esta región parece efectivamente haber sido diversa y que, además de involucrar a distintos grupos especializados, se configuró un entramado complejo de redes de interacciones ocasionales y/o programadas entre formaciones sociales específicas, así como entre diferentes individuos. Más precisamente, pensamos que las interacciones fueron variando a lo largo del Arcaico en cuanto a direccionalidad, distancia, velocidad, frecuencia, ritmo e intensidad, ampliando las propuestas clásicas que enfatizan la necesidad de moverse en búsqueda de alimentos y analizan la movilidad solo con base en las estrategias tecnológicas (Bamforth 1986; Bettinger 1981; Chatters 1987). A lo largo del Arcaico, estas interacciones involucrarían entidades sociales y actores cada vez más especializados y diferenciados, de modo de instalar las bases de lo que se ha definido como una mayor complejidad social característica del período Formativo posterior, a partir de 3.700 a 3.500 años a.p. En consecuencia, proponemos que las interacciones, materializadas por la circulación de ciertos objetos o materias primas, habrían contribuido a configurar y mantener relaciones sociales no solo a nivel intergrupos (Renfrew 1993; Webb 1974), sino también intragrupos con una mayor diferenciación social entre los individuos (Aldenferfer 1989; Close 2000), evidenciada por el acceso a recursos y bienes ornamentales personales distintivos. Ampliando entonces los clásicos conceptos de patrones, modelos o estrategias de movilidad para el extremo norte de Chile en el área Centro-Sur andino, proponemos en este trabajo inicial destacar y discutir las interacciones sociales e individuales, basándonos en el registro de objetos "misceláneos".

Para abordar las dinámicas sociales cotidianas de los cazadores recolectores altoandinos, nos centramos específicamente en el estudio de contextos arqueológicos excavados en la precordillera de Arica (2.800-3.800 msm), que corresponden a contextos de ocupaciones domésticas logísticas (Osorio et al. 2016). Ordenamos de manera sistemática la presencia de objetos "misceláneos", para evaluar su(s) permanencia(s) y cambio(s) a lo largo del período Arcaico (10.500-3.700 años a.p.). De modo de discutir más precisamente estos aspectos, se sintetizan en este trabajo nuevas dataciones obtenidas de los contextos arcaicos altoandinos estudiados.

\section{Contexto de Estudio}

El período Arcaico en las tierras altas del extremo norte de Chile consta actualmente de tres fases: Temprana (10.500-8.000 años a.p), Media (8.000-6.000 años a.p) y Tardía (6.0003.700 años a.p.). Cada fase fue caracterizada inicialmente por el análisis tipológico del material lítico, particularmente de las puntas de proyectil (Núñez y Santoro 1988; Santoro 1989; Santoro y Chacama 1982, 1984; Santoro et al. 2016). Desde hace algunos años, diversos trabajos han propuesto la revisión de esta secuencia material a partir de una aproximación tecnológica del material lítico (Herrera et al. 2015; Llanos 2015; Osorio 2012; Osorio et al. 2011; Osorio et al. 2016). No obstante, se mantienen vigente las tres fases arcaicas, pese a la existencia de otras secuencias cronológicas como las propuestas en el sur del Perú, a partir de los trabajos realizados en el sitio de Asana (Aldenderfer 1990; Aldenderfer y Flores 2011). Los cazadores recolectores altoandinos han sido definidos como grupos de baja demografía, móviles y altamente especializados en el manejo del medio ambiente de altura (Aldenderfer 1998; Llanos 2015; Núñez y Santoro 1988; Osorio 2012; Osorio et al. 2017; Santoro 1989; Santoro et al. 2016; Sepúlveda, Valenzuela et al. 2013; Sepúlveda, García et al. 2013). Las evidencias óseas y líticas los caracterizan como cazadores de camélidos y otros mamíferos más pequeños (Castillo et al. 2015; Llanos 2015; Osorio et al. 2016; Osorio et al. 2017; Santoro 1989), mientras que las estrategias tecnológicas y el aprovisionamiento en materias primas líticas dan cuenta del conocimiento especializado del entorno y una movilidad evidenciada por el uso logístico y estacional de los aleros, sitios esencialmente estudiados hasta ahora (García y Sepúlveda 2011; Osorio et al. 2016; Santoro et al. 2016; Sepúlveda, García et al. 2013). Estas características permiten distinguir a las poblaciones de los grupos cazadores recolectores y pescadores costeros, cuyos principales vestigios son funerarios y dan 
cuenta de la especialización en la explotación del ambiente marino y el uso de recursos del litoral (Arriaza et al. 2008; Marquet et al. 2012; Santoro et al. 2016; Standen et al. 2004; entre otros). Si bien sus principales asentamientos se localizan en el borde costero, estos grupos accederían esporádicamente hacia los valles interiores para la obtención de ciertas materias primas (Muñoz y Arriaza 2006; Niemeyer y Schiappacasse 1964; Schiappacasse y Niemeyer 1975; Standen y Núñez 1984), complementado posiblemente con la obtención de ciertos recursos alimenticios. En suma, hasta ahora, se han definido y distinguido dos entidades sociales asentadas en distintos pisos ecológicos y diferenciados por su especialización en el manejo de los recursos obtenibles en sus respectivos ambientes.

\section{Metodología}

En este trabajo sistematizamos contextual y cronológicamente 43 objetos procedentes de 10 sitios con ocupaciones arcaicas de las tierras altas de Arica: Los Dolmenes, Pampa El Muerto 15, Piñuta, Puxuma 1, Puxuma 2, Tangani 1, Las Cuevas, Patapatane, Tojo-tojone, Hakenasa (Figura 1). Dichos objetos se encuentran en el Museo Universidad de Tarapacá San Miguel de Azapa. Estas colecciones se conformaron a partir de excavaciones efectuadas entre los años 1970 y 1990, por lo que su obtención se logró mediante distintas metodologías de excavación y separación, así como de diferentes problemáticas y objetivos de investigación. A partir de la información completa de los sitios, se observa la recuperación de pocos objetos en sitios más intensa y extensivamente excavados, al contrario, en aquellos con acotado depósito ocupacional se registra una mayor cantidad de objetos. En consecuencia, la cantidad de registro no pareciera responder al volumen de sedimentos excavados, sino a las actividades desarrolladas al interior de los sitios, las que claramente no se restringieron a la manufactura de herramientas líticas y el destazamiento y consumo de animales. Por ello, pese a que el registro arqueológico pareciera poco comparable, consideramos viable su utilización para los fines de la discusión propuesta.

Para la caracterización de cada objeto se consignó el contexto arqueológico de origen y se precisó la cronología atribuida a dicho contexto. Para ello se complementaron las dataciones ya existentes para los sitios estudiados con nuevas dataciones radiocarbónicas especificadas en las Tablas 1 y 2 . Hasta este estudio, salvo en el caso de Hakenasa y Patapatane, la mayoría de los sitios altoandinos consignaban solo una fecha, generalmente relacionada con el estrato de mayor intensidad ocupacional. De modo de precisar las secuencias ocupacionales de los sitios y su función, procuramos ampliar la cantidad de dataciones disponibles. Se escogieron así niveles al inicio y final de las ocupaciones (estratos bases y superiores, respectivamente), así como intermedios (Tablas 1 y 2).

\section{Resultados}

A la fecha se identificaron 43 objetos "misceláneos" en los sitios seleccionados para este estudio, los cuales fueron descritos a partir de su morfología general, aunque algunos se encontraron fragmentados. El sitio con mayor cantidad es Patapatane $(n=18)$, mientras que Los Dolmenes, Puxuma 2, y Las Cuevas presentan solo un registro cada uno.

Se registraron 35 objetos ornamentales y/o suntuarios de origen costero, de ellos 31 corresponden a fragmentos de conchas, dos corresponden a vértebras de pescado, un diente de escualo (completo) y un collar de cuentas de Olivia peruviana, reportados en siete de los diez sitios analizados. Las conchas en su mayoría corresponden a fragmentos de Choromitylus chorus y han sido reportadas en los sitios Pampa El Muerto 15, Puxuma 1, Puxuma 2, Tangani 1, Patapatane y Tojo-Tojone, entre 3.000 y $3.800 \mathrm{msm}$ (Figura 2, I.a a I.c). Estos fragmentos de valvas no presentan evidencias de filos o modificaciones que indiquen su uso como herramientas de corte, además que son de tamaño pequeño (inferior a 5 $\mathrm{cm}$ ). El diente de escualo proviene de los estratos tempranos del sitio Las Cuevas, situado por sobre $4.000 \mathrm{msm}$.

Los sitios Patapatane y Puxuma 2, los únicos sitios con contextos funerarios de la precordillera, contienen evidencias costeras: vértebras de pescado y fragmentos de Choromytilus chorus en Patapatane y un collar de cuentas de Olivia peruviana en Puxuma 2. Dichos contextos permiten dar cuenta de la particular valoración de estos objetos. Similarmente, el diente de escualo reportado en Las Cuevas, interpretado como elemento suntuario, al no existir otras evidencias que den cuenta del consumo del 


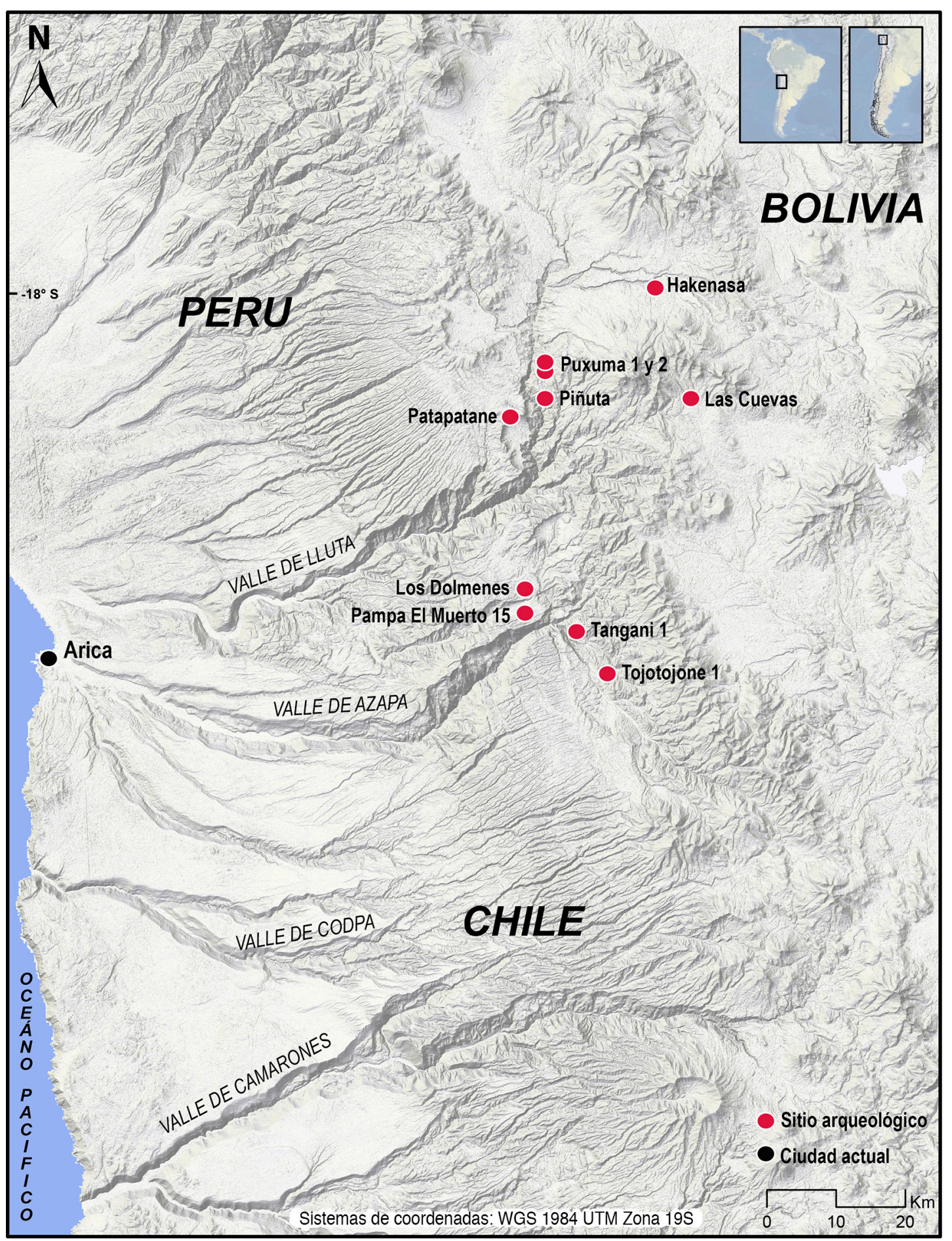

Figura 1. Mapa con los sitios presentados en el análisis.

Maps with the location of sites analysed. 
I

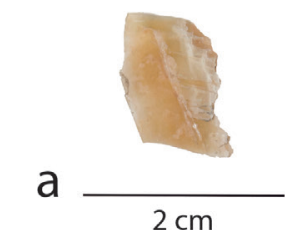

II

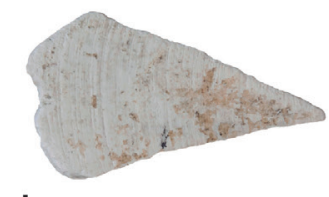

b

$2 \mathrm{~cm}$
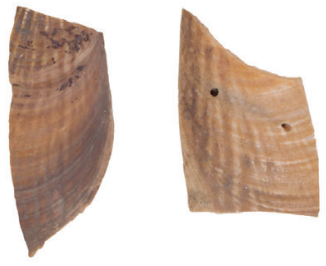

C $\frac{}{2 \mathrm{~cm}}$

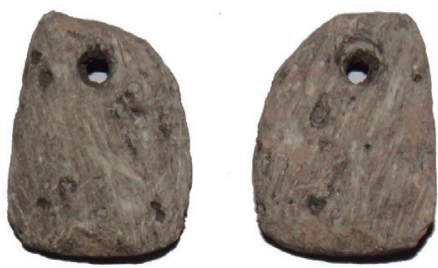

C

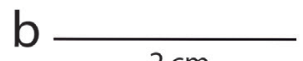

$2 \mathrm{~cm}$

III

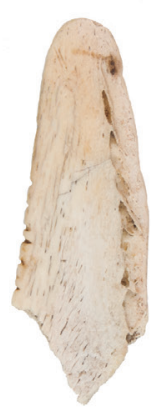

a $2 \mathrm{~cm}$

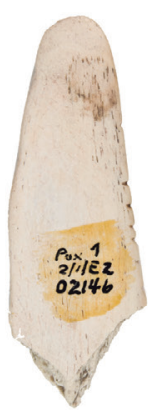

Paxiez
20.112
02146

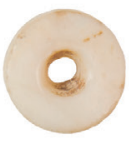

$\mathrm{cm}$

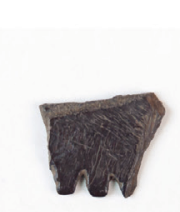

b

$2 \mathrm{~cm}$

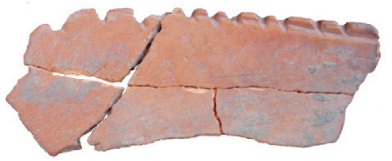

C

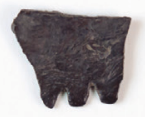

-

\section{IV}

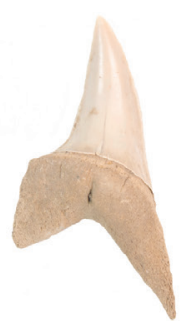

$2 \mathrm{~cm}$

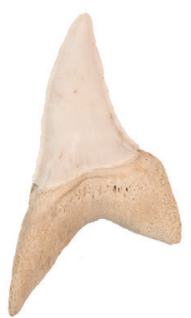

a

$$
2 \mathrm{~cm}
$$

Figura 2. Ejemplos de objetos misceláneos. (I) Fragmentos de conchas de (I.a) Mytilidae en Pampa El Muerto 15; (I.b) Choromytilus chorus en Puxuma 1; (I.c) Mytilidae en Tangani 1; (II) Cuentas y colgantes (II.a) Los Dolmenes; (II.b) Puxuma 1; (II.c) Tangani 1; (III) Fragmentos óseos con incisiones (III.a) Puxuma 1; (III.b) Piñuta; (III.c) Pampa El Muerto 3; (IV) Diente de escualo (Lamnidae Isurus sp.) Alero El Bajo.

Examples of miscellaneous objects (I) Shell fragments (I.a) Mytilidae from Pampa El Muerto 15; (I.b) Choromytilus chorus from Puxuma 1; (I.c) Mytilidae from Tangani 1; (II) Beads and pendants (II.a) Los Dolmenes; (II.b) Puxuma 1; (II.c) Tangani 1; (III) Bone fragments wiht incisions (III.a) Puxuma 1; (III.b) Piñuta; (III.c) Pampa El Muerto 3; (IV) Tooth of shark (Lamnidae Isurus sp.) Alero El Bajo. 
animal in situ, permite hipotetizar sobre la valoración otorgada a este tipo de objeto. El reciente hallazgo de otro ejemplar similar en el sitio El Bajo, sector de Mullipungo, también posiblemente del Arcaico Temprano, incide en la valoración de este tipo de objetos por parte de los cazadores recolectores altoandinos (Sepúlveda, comunicación personal 2015; Figura 2, IV.a).

Los objetos ornamentales con materias primas locales son ocho, predominando la presencia de cuentas de collar en tres de los 10 sitios en estudio: Los Dolmenes, Tangani 1, Hakenasa (Figura 2, II.a a I.c; Tabla 1). La mayoría de los elementos corresponden a cuentas manufacturadas en hueso o en piedra. Además de las cuentas se consignan: un fragmento de hueso con surcados en su borde en Piñuta y un fragmento similar con borde denticulado y superficie pulida en el sitio Puxuma 1 (Figura 2, III.a y III.b), semejante a otro reportado para el lapso temporal correspondiente al Formativo en Pampa El Muerto 3 (Figura 2, III.c; Sepúlveda, García et al. 2013).

En términos cronológicos notamos un evidente incremento de objetos "misceláneos" hacia la fase tardía del Arcaico $(n=20)$. Esto coincide con el hecho que la mayoría de los contextos analizados se concentran en esta fase, coincidiendo con una mayor intensidad ocupacional de los pisos altoandinos en esta época (Sepúlveda, Valenzuela et al. 2013). Del total de objetos hallados, la mayor cantidad de procedencia foránea a las tierras altas se consigna en los contextos arcaicos tardíos $(n=14)$. Mientras que para la fase media el registro se reduce a cinco fragmentos de valvas en Patapatane y Pampa El Muerto 15. Finalmente, para la fase temprana el registro es sumamente acotado y se remite a un diente de escualo en el sitio Las Cuevas. En el caso de los objetos "misceláneos" de procedencia local, en los contextos del Arcaico tardío se contabilizaron cinco objetos, correspondientes a cuentas de collar, un fragmento plano de hueso con borde surcado en Puxuma 1 y un fragmento plano de hueso pulido también con borde denticulado en Piñuta. Para el Arcaico Medio no se consignaron objetos, mientras para la fase temprana solo se registran tres cuentas óseas en Hakenasa.

Por último, para los contextos con fechas posteriores a 3.700 años a.p., coincidente con el Formativo en el área Centro Sur Andina, se registraron quince objetos de origen costero en los sitios Tojo Tojone, Puxuma 2 y Patapatane (Tabla 2).

\section{Objetos "Misceláneos" a lo Largo del Arcaico}

Entre los objetos "misceláneos", los de procedencia costera otorgan y precisan un nivel de información relacionado con el origen de los materiales y por ende, la direccionalidad de los movimientos identificados. También permiten discutir el tipo de mecanismo de interacción con otros grupos sociales, especializados y asentados en distintos pisos ecológicos, a lo largo del tiempo.

Para el Arcaico Temprano, los modelos de movilidad existentes sugieren que los cazadores recolectores altoandinos se habrían desplazado articulando diferentes espacios, entre estos, la costa (Núñez y Santoro 1989; Santoro 1993; Santoro y Chacama 1982 y 1984). Dichos movimientos pudieron estar orientados al reconocimiento del medio ambiente y la distribución de los recursos fundamentales para la subsistencia. Así también, pudieron establecerse los primeros contactos entre diferentes entidades sociales, posteriormente reforzados mediante relaciones más constantes. De modo similar al norte de Chile, Aldenderfer (1989, 1999) a partir de sus trabajos en Asana, sierra sur de Perú, destaca una movilidad entre los pisos altoandinos y otros situados hacia el litoral Pacífico. De acuerdo al autor, en un comienzo mediante movimientos exploratorios se tomaría contacto con otros grupos, generando el flujo de pocos objetos referidos principalmente a la esfera de lo económico (Aldenderfer 1999). En las tierras altas de Arica, pensamos que pudo haberse desarrollado una conexión similar, aunque conjuntamente con la circulación de objetos o materias primas foráneas, no necesariamente ligados a su consumo alimenticio, lo que explicaría las evidencias malacológicas presentes en Pampa El Muerto 15, Patapatane y Las Cuevas, en estos momentos del Arcaico. La cantidad de objetos y sus pequeños tamaños hacen pensar que su circulación fue ocasional. A la inversa, la presencia escasa de manganeso proveniente de áreas de aprovisionamiento situadas por sobre $4.500 \mathrm{msm}$ (Sepúlveda, Valenzuela et al. 2013) en contextos costeros contemporáneos, permite ahondar en la existencia de tempranos intercambios de materias primas específicas con grupos altoandinos.

A la fecha, no existen evidencias de objetos "misceláneos" de procedencia costera en los contextos precordilleranos para la fase Arcaico Medio. Durante el lapso temporal que abarca entre los 8.000 y 6.000 


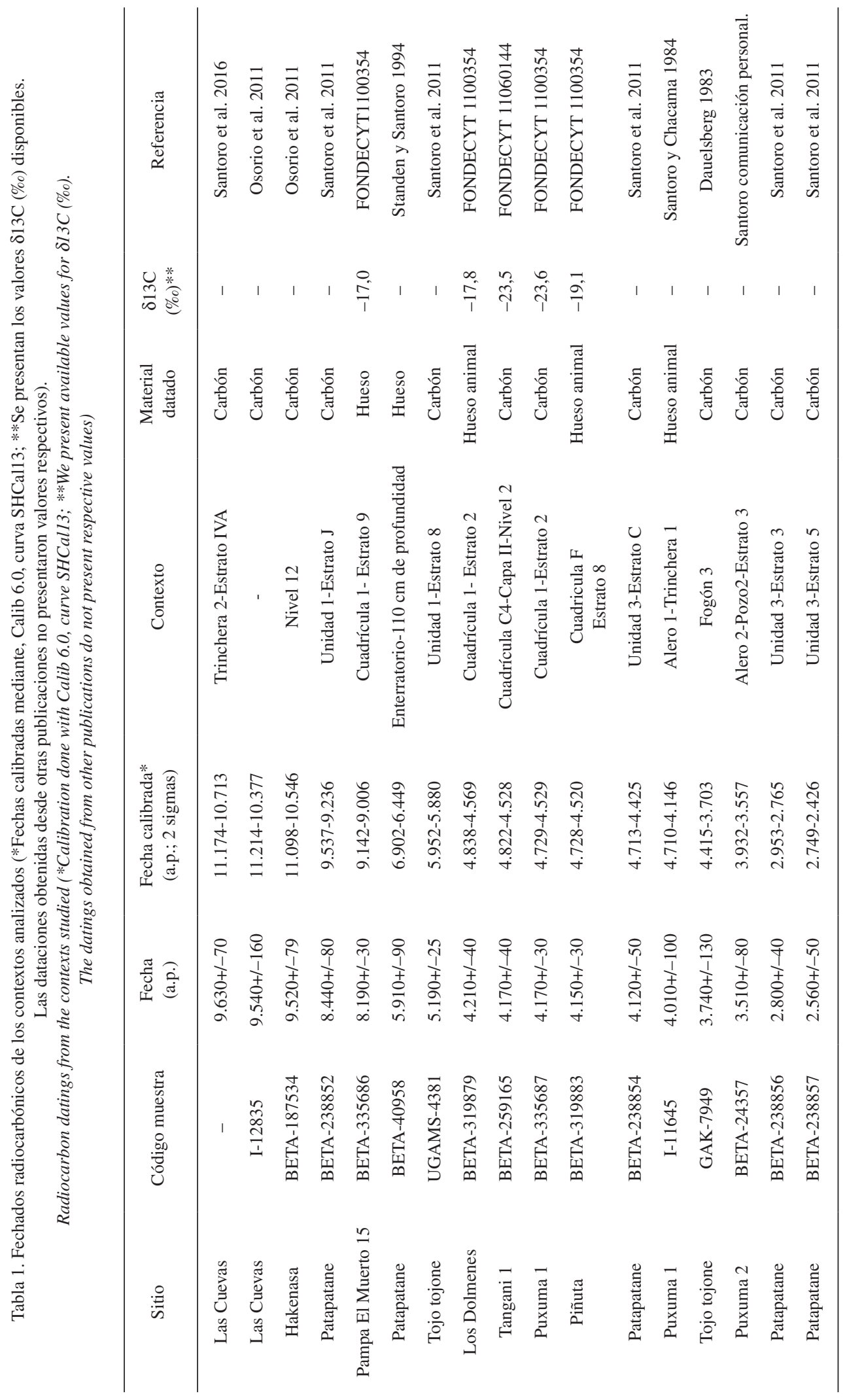




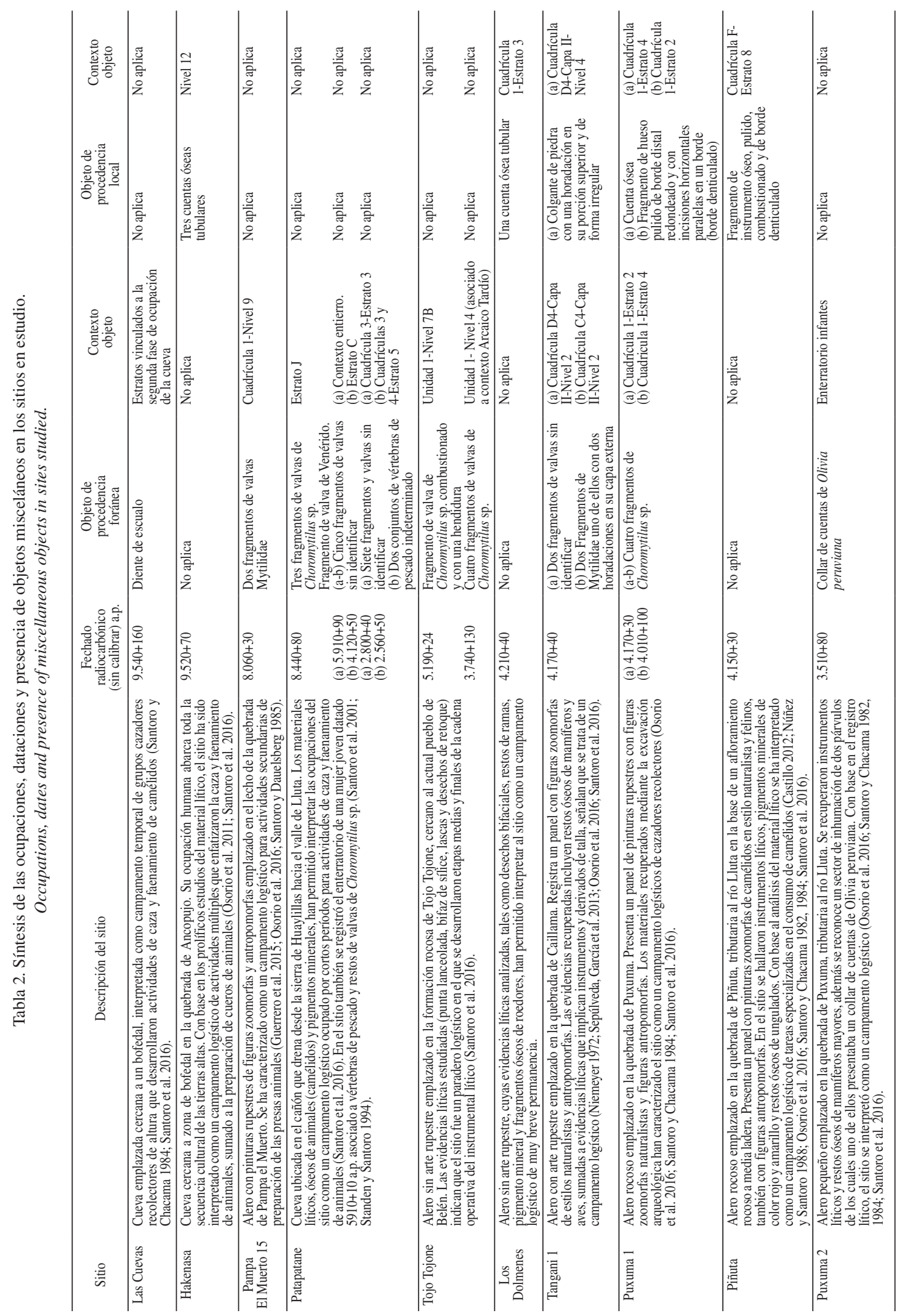


años a.p. las evidencias ocupacionales en la zona se reducen considerablemente, no obstante recientes hallazgos en evaluación nos permitirán sin dudas re-evaluar las características del asentamiento altoandino para esta fase. A diferencia, en la costa se incrementan los contextos de cazadores recolectores pescadores para este lapso temporal (Standen et al. 2004; Standen et al. 2014), varios de ellos con la vigencia del uso de manganeso procedente de tierras altas (Sepúlveda, Valenzuela et al. 2013). Así, se mantendrían procesos de intercambio entre grupos costeros y altoandinos, pese a que estos últimos modificaran su patrón de asentamiento y movilidad en este tiempo. Durante las fases tempranas y medias, los grupos altoandinos mantendrían una movilidad estacional entre los distintos pisos situados por sobre los $3.000 \mathrm{msm}$, complementado con movimientos norte-sur como lo indican similitudes en las tipologías líticas entre el norte de Chile y el sur del Perú (Klarich y Aldenderfer 2005). Junto a ello, ocurrirían intercambios ocasionales con grupos diferenciados y especializados de la costa

A medida que transitamos hacia la fase tardía del Arcaico, los modelos de movilidad propuestos para este tiempo precisan circuitos más acotados y restringidos (costa y valles bajos/precordillera y puna) (Núñez y Santoro 1989; Santoro 1993). En estos momentos, el arte rupestre de Tradición Naturalista común en la sierra sur del Perú y la precordillera del norte de Chile, en una cota promedio situada entre 3.000 y $3.800 \mathrm{msm}$, indicaría un flujo de información visual y la creación de un paisaje cultural y territorio compartido por grupos móviles que mantendrían un énfasis cazador recolector, en relación a los cambios socioeconómicos ocurridos a partir de 6.000 años a.p. en las demás regiones del área Centro Sur andina (Sepúlveda, García et al. 2013; Sepúlveda et al. 2017). Estos movimientos serían complementados, no obstante, por interacciones esporádicas, aunque más permanentes, con las entidades sociales asentadas en la costa y valles bajos, lo que habría contribuido a la circulación de objetos "misceláneos", presentes en mayor cantidad en esta fase, tal como lo evidencian los contextos de la precordillera.

\section{Objetos "misceláneos": producción, circulación y conformación de redes sociales}

La producción y/u obtención de estos objetos es indisociable de las prácticas de caza y recolección para nuestro caso de estudio y, si bien representan esferas diferentes a la subsistencia, no existen elementos que permitan aislarlos de estas prácticas significativas en la vida cotidiana de las sociedades altoandinas.

Los objetos "misceláneos" están destinados a perdurar en el tiempo (Helms 1993), así como a circular entre diferentes lugares y/o personas, representando de paso las obligaciones sociales de las cuales están investidos (Gallardo 2016; Helms 1993; Lam 2012). De aquí que se derive una de sus características fundamentales: su capacidad para moverse a través de largas distancias espaciales (viaje que realiza el objeto), temporales (carga ancestral que puede poseer) y sociales (carga simbólica que representa en la estructuración social). Estas características los transforman en elementos fundamentales al momento de materializar relaciones sociales (Eriksen 2002; Goldstein 2000; Helms 1993; Renfrew 1993; Webb 1974; Whallon 2006) y políticas (Gallardo 2016; Yacobaccio 2012). Adicionalmente, la circulación de objetos "misceláneos" consolida un conjunto de mecanismos de interacción entre entidades sociales con especializaciones diferentes e individuos puesto que, además de dar y recibir algo a cambio, implica establecer relaciones intergrupales e interpersonales constantes (Aldenderfer 1989; Helms 1993, Reynolds et al. 2003; Whallon 2006).

Una red social entendemos se refiere a grupos de personas que cooperan en la manipulación de los recursos y establecen acuerdos y reglas para su obtención, circulación y uso. De esta manera se asegura el acceso a recursos de subsistencia y utilitarios, a la vez que permite la circulación de información, ideas e ideologías (Fitzhugh et al. 2011; Hamilton et al. 2007; Loponte et al. 2004). Las redes se materializan, entre otras formas, mediante la circulación e intercambio de objetos (primordialmente regalos) que simbolizan de paso la adscripción a dicha red (Reynolds et al. 2003; Schortman y Urban 2012; Whallon 2006). Constituyen procesos cooperativos de agencia colectiva, cuyo fin primordial va más allá del intercambio de recursos de subsistencia. Además, contribuyen al establecimiento de marcadores culturales que permiten el flujo de información e ideologías y consolidan diferentes segmentos de la estructura social al interior de los grupos (Aubry et al. 2012; Borrero et al. 2011; Fuenzalida y Gallardo 2013; Goldstein 2000; Munson y Macri 2009). 
La circulación de objetos e información se añade a las prácticas de caza y recolección, actividades que fueron indudablemente un objetivo central de las dinámicas sociales de las antiguas poblaciones, pero que se encuentran a su vez embedded del flujo de ideas y consecuentemente de la circulación de objetos con una carga social (Whallon 2006).

La circulación de objetos "misceláneos" constituye un elemento central para evidenciar la interacción entre grupos sociales, así como al interior de dichas entidades, pues implica hallar determinados objetos foráneos en contextos locales (Aldenderfer 2008; Eriksen 2002; Gallardo 2016; Hamilton et al. 2007; Helms 1993; Whallon 2006; Yacobaccio 2006 y 2012). Estos objetos reflejan expresiones sociales y comunicacionales entre grupos que pueden presentar especializaciones económicas diferentes (Aubry et al. 2012; Eriksen 2002, Yacobaccio 2006; Whallon 2006). En algunos casos, dichos objetos pueden llegar a constituir elementos identitarios de cada grupo (Fitzhugh et al. 2011; McDonald y Veth 2011; Wiessner 1982). En otros, pueden ser las representaciones materiales de los segmentos que se ven involucrados en las redes sociales y el estatus que poseen dentro del grupo (Appadurai 1986; Bender 1978; Eriksen 2002; Fitzhugh et al. 2011; Fuenzalida y Gallardo 2013; Gallardo y Cabello 2015; Hamilton et al. 2007; Yacobaccio 2006 y 2007).

En consecuencia, la explotación de distintas materias primas y producción diferencial de objetos "misceláneos" por parte de grupos diferenciados y especializados sumado a diferentes tipos de demandas, no solo puede ser interpretado en relación la movilidad de grupos distintos y al intercambios y flujo de objetos a lo largo del Arcaico, sino que pueden contribuir a comprender la generación de redes de interacción necesarias entre distintas entidades sociales, con la instauración de deberes y obligaciones entre ellos.

En el extremo norte de Chile la valoración de ciertas materias primas u objetos acabados, pudo ser un motor para la generación de mecanismos de intercambio entre grupos especializados y diferenciados. El origen costero de la mayoría de los objetos "misceláneos" presentes en la precordillera permite en efecto reflexionar sobre su valorización y por ende en la generación de demanda por parte de los grupos de tierras altas. De la misma forma, materiales originarios de tierras altas, como manganeso en los cuerpos momificados Chinchorro (Sepúlveda,
Valenzuela et al. 2013), restos de obsidiana en el sitio Acha 2 (Muñoz y Chacama 1993) y piel de vicuña en el sitio Camarones 14 (Schiappacasse y Niemeyer 1984) permiten contrastar las demandas generadas por estas distintas entidades sociales. A diferencia de la costa, el poco uso de óxido de manganeso en los contextos arcaicos de tierras altas, además de razones relacionadas con su uso o función simbólica (Sepúlveda, Valenzuela et al. 2013), puede también ser comprendido desde la distinta valoración que le fue otorgado por los grupos altoandinos. No obstante, la localización de las áreas de aprovisionamiento del manganeso a $4.500 \mathrm{msm}$ hace pensar que fueron ellos los encargados de su explotación, para luego construir y sustentar variadas formas de interacción con los grupos costeros.

La producción y circulación de objetos "misceláneos" habría estado definida por el valor que les fue atribuido, con la necesaria y relativa producción de excedentes por parte de los distintos grupos arcaicos de modo de alimentar intercambios e interacción, mecanismos similares a los observados en otras regiones del norte de Chile como en la cuenca del río Loa y la costa de Antofagasta (Ballester y Gallardo 2011) y en el Salar de Atacama (Núñez et al. 2006). Durante los intercambios ocurrirían flujos de información necesarios entre las distintas entidades sociales involucradas mediante la circulación de objetos con cargas simbólicas específicas.

\section{Objetos “misceláneos” y diferenciación social intragrupo}

En la mayoría de los casos, los objetos "misceláneos" corresponden a ornamentos corporales u objetos suntuarios (Santoro y Chacama 1984; Standen y Santoro 1994). Su escasez puede ser comprendida por su acceso restringido a ciertos individuos, pudiendo ser interpretado por la diferenciación existente entre los individuos al interior de los grupos (Appadurai 1986; Helms 1993). La acotada cantidad de cuentas de collar en los sitios de Hakenasa, Los Dolmenes y Puxuma 1, sumado al colgante de piedra de Tangani 1 y la presencia de huesos de borde denticulado en Piñuta y Puxuma 1, es acorde con una obtención restringida de este tipo de objetos ornamentales entre los cazadores recolectores altoandinos. Asimismo, en los únicos dos contextos funerarios registrados hasta el momento en la precordillera se 
han hallado ornamentos corporales elaborados con conchas de la costa pacífica: valva de Choromytilus chorus en la cueva de Patapatane junto a tres huesos de pescado sobre el cuerpo de una mujer adulta inhumada (Santoro et al. 2001; Standen y Santoro 1994); cuentas de Olivia peruviana en el alero Puxuma 2 sobre uno de los párvulos enterrados en el sitio (Santoro y Chacama 1984). El registrar estos objetos en este tipo de contexto refuerza el carácter simbólico y su particular valorización para los cazadores recolectores altoandinos.

Su mayor cantidad paralela al gradual aumento de la complejización social a lo largo del Arcaico, pueden ser comprendidos en relación con la intensificación de los procesos de diferenciación social entre grupos, pero también entre individuos (Appadurai 1986; Fitzhugh et al. 2011; Helms 1993; Munson y Macri 2009; Yacobaccio 2001). La complejización social a la que nos referimos estaría orientada a la constante reestructuración de las relaciones de los grupos arcaicos, proceso que se evidencia con mayor precisión y cantidad de datos hacia los 6.000 y 5.000 años a.p. Por ejemplo, en la costa, el aumento demográfico y una mayor diferenciación social, sumada a su especialización económica, se manifestó en el desarrollo y aumento de las prácticas de momificación intencional en la Tradición Cultural Chinchorro (Arriaza 1995; Arriaza et al. 2005 y 2008; Núñez y Santoro 2011; Marquet et al. 2012; Sepúlveda, Valenzuela et al. 2013; Standen et al. 2004, Standen et al. 2014). La mayor variabilidad en los tipos de preparación mortuoria, pensamos, puede ser interpretado como el acceso diferencial a los recursos y materias primas empleados en cada caso y, por ende, como una mayor diferenciación social entre individuos al interior de un mismo grupo cultural.

Esto nos permite plantear que los procesos de circulación y/o intercambio de objetos "misceláneos" participaron sin dudas en la construcción y sostenimiento de la complejización social posteriormente consolidado en el período Formativo (Núñez y Santoro 2011; Yacobaccio 2012), transformando de paso los mecanismos de producción, circulación y distribución entre distintas entidades sociales, pero también entre individuos.

\section{Palabras Finales}

La cantidad y tipo de objetos "misceláneos" evidenciados, hasta ahora, en los contextos arcaicos de la precordillera del extremo norte de Chile nos informan exploratoriamente de la existencia de distintas dinámicas e interacciones sociales, las que probablemente fueron variando e intensificándose en el tiempo. La mayor cantidad de objetos junto con la mayor intensidad ocupacional de este espacio hacia fines del Arcaico dan cuenta cómo se fueron transformando y estructurando nuevas formas de movilidad y redes de interacción. En ellas, los objetos misceláneos fueron adquiriendo protagonismo de modo que además de contribuir en las relaciones entre grupos diferentes, permitieron distinguir a los individuos en su interior. En ese contexto, junto al arte rupestre, fueron configurando en las tierras altas del extremo norte de Chile una historia común, diferenciada de lo acontecido en otras regiones, como la costa o el altiplano, pero en ningún caso separado o marginado. Todas estas regiones interactuaron constantemente en el interior de complejas y variadas redes o entramados de interacción económica, pero también social.

La presencia de objetos "misceláneos" en los contextos arcaicos precordilleranos nos permite reinsertar este espacio en los procesos consolidados posteriormente en el Formativo. La precordillera con sus particularidades fisiográficas y ambientales, pensamos, resultó ser un espacio clave en relación con el desarrollo del pastoreo como actividad económica de gran relevancia para las distintas sociedades formativas de la región Centro Sur Andina. Aquello debió generar la instauración de mecanismos de interacción necesarios para negociar su uso, frente a grupos cazadores recolectores que aparentemente siguieron manteniendo su modo de vida hasta los primeros siglos de nuestra era (Sepúlveda et al. 2017). Estos mecanismos involucraron instancias simbólicas estrechamente relacionadas con las prácticas cotidianas de subsistencia de los cazadores recolectores altoandinos, como lo sugiere el arte rupestre en el cual se observan cambios escénicos importantes para estos momentos (Dudognon y Sepúlveda 2016). Sin abandonar la caza, las escenas de capturas de animales en vida, así como su protección, las figuras humanas a modo de pastores, individualizados y ataviados con tocados y objetos en sus manos, irrumpen significativamente en los paneles pintados de la precordillera.

Los materiales que se mueven y que poseen un carácter más bien simbólico, ornamental, político o ideológico no dejan de ser fundamentales 
para la supervivencia física de los cazadores recolectores, pues desempeñan un rol importante en la configuración de las relaciones sociales inter e intragrupales (Fitzhugh et al. 2011; Hamilton et al. 2007). Permiten establecer y sostener mecanismos de contacto e interacción social en distintos niveles de distancia espacial y social, además aseguran el flujo de información e integran la esfera ideológica en la organización social de estos grupos (Fitzhugh et al. 2011; Hamilton et al. 2007; Whallon 2006). Por ello, el estudio de los objetos "misceláneos" resulta fundamental en la medida que materializan también aspectos ideológicos de la organización social (Appadurai 1986:38; Aubry et al. 2012), más aún para las sociedades cazadores recolectores altoandinas en las que estos aspectos son raramente discutidos.

Estas hipótesis han de ser contrastadas con una mayor resolución de datos que integre los antecedentes procedentes de nuevos contextos arcaicos aún en estudio, además de la incorporación de otras líneas de evidencia en estas interpretaciones. Recientes contextos arcaicos en la precordillera han aumentado la cantidad de objetos "misceláneos" reportados, lo que permitirá precisar las propuestas planteadas en este trabajo.
Explorar los aspectos sociales de los cazadores recolectores mediante los objetos "misceláneos" y su circulación durante el período Arcaico, nos orienta hacia nuevas preguntas relacionadas con las dinámicas e interacciones sociales de los cazadores recolectores altoandinos, aspectos que esperamos seguir explorando.

Agradecimientos: Los análisis desarrollados en este trabajo se iniciaron en el marco del proyecto FONDECYT 1100354 para ser complementados y finalizados en el FONDECYT 1130808. Se agradece a las Becas CONICYT de Magíster Nacional año académico 2015, Folio 22150457 y Beca Magíster en Antropología de la Universidad de Tarapacá, así como el apoyo del Convenio de Desempeño Universidad de Tarapacá-MINEDUC. También agradecemos al Museo Arqueológico San Miguel de Azapa, de la Universidad de Tarapacá por el acceso a las valiosas colecciones aquí analizadas. Permisos del CMN ns 2202, 4380, 5634, 0065, 002404, 3548, 001600. Finalmente reconocemos los acertados comentarios de Daniela Osorio, Salomon Hocsman, Benjamín Ballester, así como a los evaluadores anónimos quienes contribuyeron a mejorar sustancialmente este trabajo.

\section{Referencias Citadas}

Aldenderfer, M. 1989. The Archaic Period in the South-Central Andes. Journal of World Prehistory 3:117-158.

Aldenderfer, M. 1998. Montane Foragers. Asana and the SouthCentral Andean Archaic. University of IowaPress, Iowa City.

Aldenderfer, M. 1999. Cronología y conexiones: Evidencias precerámicas de Asana. Boletín de Arqueología PUCP 3:375-391.

Aldenderfer, M. 2008. High elevation Foraging societies. En Handbook of South American Archaeology, editado por H. Silverman y W. Isbell, pp. 131-143. University of Illinois at Urbana-Champaign, Urbana.

Aldenderfer, M. y L. Flores Blanco 2011. Reflexiones para avanzar en los estudios del período Arcaico en los Andes CentroSur. Chungara Revista de Antropología Chilena 43:531-550.

Appadurai, A. 1986. Introduction: commodities and the politics of values. En The Social Life of things. Commodities in Cultural Perspectives, editado por A. Appadurai, pp. 3-63. Cambridge University Press, Cambridge.

Arriaza, B., M. Doubrava, V. Standen y H. Haas 2005. Differential mortuary treatment among the Andean Chinchorro fishers: Social inequalities or in situ regional cultural evolution? Current Anthropology 46:662-671.
Arriaza, B., V. Standen, V. Cassman y C. Santoro 2008. Chinchorro Culture: Pioneers of the coast of the Atacama Desert. En Handbook of South-American Archaeology, editado por H. Silverman y W. Isbell, pp. 45-58. University of Illinois at Urbana-Champaign, Urbana.

Aubry, T., L.J. Mangado y H. Matias 2012. We will be known by the tracks we leave behind: Exotic lithic raw materials, mobility and social networking among the Côa Valley foragers (Portugal). Journal of Anthropological Archaeology 3:528-550.

Ballester, B. y F. Gallardo 2011. Prehistoric and historic networks on the Atacama Desert coast (northern Chile). Antiquity 85:875-889.

Bamforth, D. 1986. Technological efficiency and tool curation. American Antiquity 51: 38-50.

Bargatzky, T. 1984. Culture, environment, and the ills of adaptationism. Current Anthropology 25: 399-415.

Bettinger, R. 2007 [1980]. Los cazadores-recolectores del Holoceno. En Clásicos de la teoría arqueológica contemporánea, editado por L. Orquera y V. Horwitz , pp. 493-563. Sociedad Argentina de antropología, Buenos Aires.

Bender, B. 1978. Gatherer-hunter to farmer: A social perspective. World Archaeology 10:204-222. 
Borrero, L., F. Martin y R. Barberena 2011. "Visits", Fueguians and information networks. En Information and Its Role in Hunter-Gatherer Bands (Ideas, Debates and Perspectives, Vol 5), editado por R. Whallon, W. Lovis y R. Hitchcock, pp. 249296. Cotsen Institute of Archaeology, University of California, Los Angeles, Santa fe, Nuevo México.

Castillo, C. 2012. Aproximación a la Movilidad e Interacción Arcaica en el Extremo Norte de Chile, a través de Objetos "No-Utilitarios". Memoria para optar al título de Arqueóloga, Universidad de Tarapacá, Arica.

Castillo, C., N. Goepfert, M. Sepúlveda y T. Saintenoy 2015. Animals in highlands: Synthesis and characterization of animal presence along cultural sequence in Arica precordillera, northern Chile. Ponencia presentada en $12^{\text {th }}$ International Conference of Archeozoology, San Rafael.

Conneller, Ch. 2004. Convertirse en venado. Transformaciones corporales en Star Carr. Archaeological Dialogues 11:37-56.

Close, A. 2000. Reconstructing movement in prehistory. Journal of Archaeological Method and Theory 7:49-77.

Chatters, J. 1987. Hunter-Gatherer adaptations and assemblage structure. Journal of Anthropological Archaeology 6:336-375.

Craig, N. 2012. Transiciones del Arcaico Tardío al Formativo Temprano. Una perspectiva desde la arqueología de la unidad doméstica en dos sitios del valle del río Ilave, cuenca del lago Titicaca. En Arqueología de la cuenca del Titicaca, Perú, editado por L. Flores Blanco y H. Tantaleán, pp. 41-130. Instituto Frances de Estudios Andinos, Lima.

Dillehay, T. y P. Kaulicke 2007. Reflexiones acerca del surgimiento del poder y orden tempranos. Boletín de arqueología PUCP 11:405-415.

Dudognon, C. y M. Sepúlveda 2016. Rock art of the upper Lluta valley, northernmost of Chile (South Central Andes): A visual approach to socio-economic changes between Archaic and Formative periods (6,000-1,500 years BP). Quaternary International, en prensa.

Eriksen, B. 2002. Fossil mollusks and exotic raw materials in late glacial and early postglacial find contexts: A complement to lithic studies. En Lithic Raw Material Economy in Late Glacial and Early Postglacial Western Europe, editado por L. Fisher y B. Eriksen, pp. 27-52. British Archaeological Reports S1093, Oxford.

Fitzhug, B., S. Colby Phillips y E. Gjesfeld 2011. Modeling variability in hunter-gatherer information networks: An archaeological case study from the Kuril Islands. En Information and Its Role in Hunter-Gatherer Bands (Ideas, Debates and Perspectives, Vol 5), editado por R. Whallon, W.A. Lovis y R.K. Hitchcock, pp. 85-115. Cotsen Institute Archaeology, University of California, Los Angeles, Santa Fe, Nuevo México.

Fuenzalida, N. y F. Gallardo 2013. Exchange and ritual funerary consumption: Late marine hunter-gatherers of the Taltal coast (Atacama Desert, northern Chile). Andean Past 11:263-281.

Gallardo, F. 2016. Arqueología de los intercambios recíprocos: Costa y oasis del río loa medio e inferior, época formativa (500 cal. A.C.-700 cal. D.C.). En Monumentos Funerarios de la Costa del Desierto de Atacama: Contribuciones al Intercambio de Bienes e Información entre Cazadores-Recolectores Marinos
(Norte de Chile), editado por F. Gallardo, B. Ballester y N. Fuenzalida. CIIR-SCHA, Santiago. En Prensa.

Gallardo, F. y G. Cabello 2015. Emblems, leadership, social interaction and early social complexity: The Ancient Formative Period (1500 BC-AD 100) in the Desert of Northern Chile. Cambridge Archaeological Journal 25:615-634.

García, M. y M. Sepúlveda 2011. Contextos vegetales asociados a los aleros pintados de la precordillera de Arica. Estudios Atacameños 41:97-108.

Goldstein, P. 2000. Exotic goods and everyday chiefs: Longdistance exchange and indigenous sociopolitical development in the South Central Andes. Latin American Antiquity 11:335-361.

Hamilton, M., B. Milne, R. Walker, O. Burguer y J. Brown 2007. The complex structure of hunther-gatherer social networks. Proceeding of the Royal Society B 274:2195-2202.

Helms, M. 1993. Crafts and the Kingly Ideal. Art, Trade and Power. University of Texas Press, Texas.

Herrera, K., P. Ugalde, D. Osorio, J. Capriles, S. Hocsman y C. Santoro 2015. Análisis tecno-tipológico de instrumentos líticos del sitio arcaico temprano Ipilla-2 en los Andes de Arica, Chile. Chungara Revista de Antropología Chilena 41:41-52.

Klink, C. y M. Aldenderfer 2005. A projectile point chronology for the South-Central Andean highlands. En Advances in Titicaca Basin Archaeology, editado por C. Stanish, A.B. Cohen y M.S. Aldenderfer, vol. 1, pp. 25-54. Cotsen Institute of Archaeology, University of California, Los Angeles.

Lam, M. 2012. The first commodity: Handaxes. Ponencia presentada en Reunión Anual de la Asociación Americana para el Avance de la Ciencia, British Columbia.

Lee, R. y E. DeVore 1968. Man the Hunter. University of Chicago, Chicago.

Lavallée, D., M. Julien, P. Béarez, A. Bolaños, M. Carré, A. Chevalier, T. Delabarde, M. Fontugne, C. Rodríguez-Loredo, L. Klaric, P. Usselmann y M. Vanhaeren 2011. Quebrada de los burros. los primeros pescadores del litoral pacífico en el extremo sur peruano. Chungara Revista de Antropología Chilena 43:333-351.

Loponte, D., A. Acosta y J. Musali 2004. Complejidad social: cazadores recolectores y horticultores en la región pampeana. En Aproximaciones Contemporáneas a la Arqueología Pampeana. Perspectivas Teóricas, Metodológicas, Analíticas y Casos de Estudio, editado por G. Martinez, M. Gutierrez, R. Curtoni, M. Berón y P. Madrid, pp. 41-60. Universidad Nacional del Centro de la Provincia de Buenos Aires, Olavarría.

Lynch, T. 1975. Algunos problemas básicos del estadio cazarecolección andina: Transhumancia. Estudios Atacameños 3:9-11.

Llanos, C. 2015. Actividades Humanas de los Cazadores Recolectores del Arcaico Tardío en la Precordillera de Arica: Análisis Tecnológico Lítico del Sitio El Alto, Sector MullipungoTignamar. Memoria para optar al título de Arqueóloga. Universidad de Tarapacá, Arica.

Marquet, P., C. Santoro, C. Latorre, V. Standen, S. Abades, M. Rivadeneira, B. Arriaza y M. Hochberg 2012. Emergence of social complexity among coastal hunter-gatherers in the Atacama Desert of northern Chile. Proceedings of the National Academy of Sciences 109:14754-14760. 
Mcdonald, J. y P. Veth 2011. Information exchange among huntergatherers of the western desert of Australia. En Information and Its Role in Hunter-Gatherer Bands (Ideas, Debates and Perspectives, Book 5), editado por R. Whallon, W. Lovis y R. Hitchcock, pp. 221233. Cotsen Institute of Archaeology, Univeristy of California. Los Angeles, Santa Fe, Nuevo México.

Munson, J. y M. Macri 2009. Sociopolitical networks interactions: a case study of the Classic Maya. Journal of Anthropological Archaeology 28:424-438.

Muñoz, I. y J. Chacama 1993. Patrón de asentamiento y cronología de Acha-2. En Acha 2 y los Orígenes del poblamiento Humano en Arica, editado por I. Muñoz, pp. 21-46. Ediciones Universidad de Tarapacá, Arica.

Muñoz, I. y B. Arriaza 2006. Momificación artificial y patrón de residencia de poblaciones Chinchorro. Indicadores de una temprana ocupación de cazadores recolectores que habitaron el desierto costero de Atacama. En El Hombre Temprano en América y sus Implicaciones en el Poblamiento de la Cuenca de México: Primer Simposio Internacional, editado por J. Jiménez, S. González, J. Pompa y Padilla y J. Ortiz, pp. 107-144. Instituto Nacional de Antropología e Historia, México, D.F.

Niemeyer, H. y V. Schiappacasse 1964. Investigaciones Arqueológicas en las Terrazas de Conanoxa, Valle de Camarones (Provincia de Tarapacá). Editorial Universidad Católica, Santiago.

Núñez, L. 1975. Dinámica de grupos precerámicos en el perfil costa-altiplano, norte de Chile. Estudios Atacameños 3:53-65.

Núñez, L. y T. Dillehay 1995 [1979]. Movilidad Giratoria, Armonía Social y Desarrollos en los Andes Meridionales: Patrones de Tráfico e Interacción Económica. Universidad Católica del Norte, Antofagasta.

Núñez, L. y C. Santoro 1988. Cazadores de la puna seca y salada del área Centro su andina (norte de Chile). Estudios Atacameños 9:13-65.

Núñez, L. y C. Santoro 2011. El tránsito arcaico-formativo en la circumpuna y valles occidentales del centro sur andino: hacia los cambios "neolíticos". Chungara Revista de Antropología Chilena 43:487-530.

Núñez, L., I. Cartajena, C. Carrasco, P. de Souza y M. Grosjean 2006. Emergencia de comunidades pastoralistas formativas en el sureste de la Puna de Atacama. Estudios Atacameños 32:93-117.

Núñez, L., V. Zlatar y P. Núñez 1975. Un circuito transhumántico entre la costa de Pisagua y el borde occidental de la Pampa de Tamarugual. Estudios Atacameños 3:45-47.

Osorio, D. 2012. Reevaluación del Arcaico Temprano de la Puna Seca (12.000 años cal. AP-9.000 años cal. AP). Implicancias para el Poblamiento Inicial del Altiplano del Norte Grande de Chile. Memoria para optar al título de Arqueóloga. Universidad de Chile, Santiago.

Osorio, D., D. Jackson, P. Ugalde, C. Latorre, R. De Pol-Holz y C. Santoro 2011. Hakenasa Cave and its relevance for the peopling of the southern Andean Altiplano. Antiquity 85:1-15.

Osorio, D., M. Sepúlveda, C. Castillo y M. Corvalán 2016. Análisis lítico y funcionalidad de sitios de los aleros de la precordillera de Arica (Centro-Sur andino) durante el período Arcaico (ca. 10.000-3.700 años A.P.). Intersecciones en Antropología 17:77-90.
Osorio, D., J. Capriles, P. Ugalde, K. Herrera, C. Salas, M. Sepúlveda, E. Gayo, C. Latorre, D. Jackson y C. Santoro 2017. Hunter-Gatherer Mobility Strategies in the High Andes of northern Chile during the late Pleistocene-early Holocene Transition (ca. 11,500-9,500 cal yr B.P.). Journal of Field Archaeology, en prensa.

Renfrew, C. 1993. Trade beyond the material. En Trade and Exchange in Prehistoric Europe, editado por C. Scarre y F. Healy, pp. 5-16. Oxbow Monographs Vol.33, Oxbow, Oxford.

Reynolds, R., T. Kholer y Z. Kobti 2003. The effects of generalized reciprocal exchange on the resilence of social networks: An example from the prehispanic Mesa Verde region. Computational and Mathematical Organization Theory 9:227-254.

Santoro, C. 1989. Antiguos cazadores de la puna (9000 a 6000 a.C). En Prehistoria. Desde sus Orígenes hasta los Albores de la Conquista, editado por J. Hidalgo, V. Schiappacasse, H. Niemeyer, C. Aldunate e I. Solimano, pp. 81-105. Editorial Andrés Bello, Santiago.

Santoro, C. 1993. Complementariedad ecológica en sociedades arcaicas del área centro sur andina. En Acha 2 y los Orígenes del Poblamiento Humano en Arica, editado por I. Muñoz, B. Arriza y A. Aufderheide, pp. 133-150. Editorial Universidad de Tarapaca, Arica.

Santoro, C. y J. Chacama 1982. Secuencia cultural de las tierras altas del área centro-sur andina. Chungara 8:33-62.

Santoro, C. y J. Chacama 1984. Secuencia de asentamientos precerámicos del extremo norte de Chile. Estudios Atacameños 7:71-84.

Santoro, C., V. Standen y B. Arriaza 2001. ¿Patrón funerario arcaico o alteración postdepositacional? El enterratorio de Patapatane en los Andes Centro Sur. Chungara Revista de Antropología Chilena 33:43-49.

Santoro, C., D. Osorio, P. Ugalde, M. Sepúlveda, I. Cartajena, V. Standen, E. Gayo, A. Maldonado, M. Rivadeneira, C. Latorre, B. Arriaza, F. Rothhammer, P. De Souza, C. Carrasco y L. Núñez 2016. Cazadores recolectores y pescadores arcaicos del desierto de Atacama. Entre el Pacífico y los Andes. Norte de Chile (ca. 10.000-3.700 A.P.). En Prehistoria en Chile: desde sus Primeros Habitantes hasta los Incas, editado por F. Falabella, M. Uribe, L. Sanhueza, C. Aldunate y J. Hidalgo, pp. 117-180. Editorial Universitaria, Santiago.

Sepúlveda, M. 2011. La tradition naturaliste des peintures rupestres des groupes chasseurs-cueilleurs de l'extrême nord du Chili. CTHS (Comité des Travaux Historiques et Scientifiques). En Préhistoires en Amérique, editado por D. Vialou, pp. 447-459. CTHS (Comité des Travaux Historiques et Scientifiques), Paris.

Sepúlveda, M., M. García, E. Calás, C. Carrasco y C. Santoro 2013. Pinturas rupestres y contextos arqueológicos de la precordillera de Arica (Extremo norte de Chile). Estudios Atacameños 46:27-46.

Sepúlveda, M., T. Saintenoy, L. Cornejo, C. Dudognon, F. Espinoza, Z. Guerrero y E. Cerrillo 2017. Rock art painting and territoriality in the precordillera of Arica, Northern Chile (South Central Andes). Archaeological and spatial approaches for the naturalistic tradition. Quaternary International. http:// dx.doi.org/10.1016/j.quaint.2017.02.005. 
Sepúlveda, M., D. Valenzuela, L. Cornejo, H. Lienqueo y H. Rousselière 2013. Óxidos de manganeso en el extremo norte de Chile: En torno al abastecimiento, producción y movilidad del color negro durante el período Arcaico. Chungara Revista de Antropología Chilena 45:143-159.

Schiappacasse, V. y H. Niemeyer 1975. Apuntes para el studio de las transhumancia en el valle de Camarones (provincial de Tarapacá, Chile). Estudios Atacameños 3:49-51.

Schiappacasse, V. y H. Niemeyer 1984. Descripción y análisis interpretativo de un sitio arcaico temprano en la quebrada de Camarones. Publicación Ocasional N ${ }^{\circ} 41$ Museo de Historia Natural, Santiago.

Schortman, E. y P. Urban 2012. Enacting power through networks. Journal of Anthropological Archaeology 31:500-514.

Standen, V. 2001. Pueblos de la costa. En Pueblos del Desierto entre el Pacífico y los Andes, editado por C. Santoro, E. Belmonte y V. Standen, pp. 29-44, Universidad de Tarapacá, Arica.

Standen, V., B. Arriaza, C. Santoro y M. Santos 2014. La práctica funeraria en el sitio Maestranza Chinchorro y el poblamiento costero durante el Arcaico Medio en el Extremo Norte de Chile. Latin American Antiquity 25:300-321.

Standen, V. y L. Núñez 1984. Indicadores antropológico-físico y culturales del cementerio precerámico Tiliviche-2 (Norte de Chile). Chungara 12:134-154.

Standen, V. y C. Santoro 1994. Patapatane 1: Temprana evidencia funeraria en los Andes de Arica (norte de Chile) y sus correlaciones. Chungara 26:165-183.
Standen, V., C. Santoro y B. Arriaza 2004. Síntesis y propuestas para el período arcaico en la costa del extremo norte de Chile. Chungara Revista de Antropología Chilena número especial Tomo I, pp. 201-212.

Webb, M. 1974. Exchange networks: prehistory. Annual Review of Anthropology 3:357-383.

Whallon, R. 2006. Social networks and information: Non"Utilitarian" mobility among hunter-gatherers. Journal of Anthropological Archaeology 25:259-270.

Wiessner, P. 1982. Risk, reciprocity and social influences on Kung San economics. En Politics and History in Band Societies, editado por E. Leacock y R. Lee, pp. 61-85. Cambridge University Press, Cambridge.

Yacobaccio, H. 2001. La domesticación de camélidos en el Noroeste Argentino. En Historia Argentina Prehispánica Vol. 1, editado por E. Berberián y A. Nielsen, pp. 7-40. Editorial Brujas, Córdoba.

Yacobaccio, H. 2006. Intensificación económica y complejidad social en cazadores-recolectores surdandinos. Boletín de Arqueología PUCP 10:305-320.

Yacobaccio, H. 2007. Población, intercambio y el origen de la complejidad social en cazadores-recolectores surandinos. En Producción y Circulación Prehispánicos de Bienes en el Surandino, compilado por A. Nielsen, M. Clara Rivolta, V. Seldes, M. Vázquez y P. Mercolli, pp. 277-287. Editorial Brujas, Córdoba.

Yacobaccio, H. 2012. Intercambio y caravanas de llamas en el sur andino. Comechingonia, Revista de Arqueología 16:13-33. 